Canad. Math. Bull. Vol. 22 (3), 1979

\title{
APPROXIMATION ON BOUNDARY SETS
}

\author{
BY \\ JAMES LI-MING WANG
}

\begin{abstract}
Let $U$ be a bounded open subset of the complex plane. By a well known result of A. M. Davie, $C(b U)$ is the uniformly-closed linear span of $A(U)$ and the powers $\left(z-z_{i}\right)^{-n}$, $n=1,2,3, \ldots$ with $z_{i}$ a point in each component of $U$. We show that if $A(U)$ is a Dirichlet algebra and $b U$ is of infinite length, then one power of $\left(z-z_{i}\right)$ is superfluous.
\end{abstract}

Let $U$ be a bounded open subset of the complex plane $\varnothing$; let $A(U)$ be the algebra of all continuous functions on $\bar{U}$ which are analytic on $U$. Let $U_{1}$, $U_{2}, \ldots$ be the components of $U$, and choose a point $z_{j} \in U_{j}$ for each $j=1$, $2, \ldots$. In [2] Davie proved that $C(b U)$ is the uniformly-closed linear span of $A(U)$ and the powers $\left(z-z_{j}\right)^{-n}, n=1,2, \ldots$, where $b U$ is the topological boundary of $U$.

If $b U_{i}$ has finite one-dimensional Hausdorff measure, then it is not hard to construct a (complex Borel) measure on $b U_{i}$ which annihilates $A(U),\left(z-z_{\mathrm{j}}\right)^{-n}$ and $\left(z-z_{i}\right)^{-(n+1)}, j \neq i, n=1,2, \ldots$ while $\int\left(z-z_{i}\right)^{-1} d \mu \neq 0$. Hence the power $\left(z-z_{i}\right)^{-1}$ is not in the closed span of $A(U)$ and other powers. The same is true for every power $\left(z-z_{i}\right)^{-n}$.

In 1957, Werner [9] observed that for every Jordan curve $\Gamma$ of infinite length, at least one power of $z$ is superfluous in spanning $C(\Gamma)$. An extension of this result has been given by Korevaar and Pfluger [6]. Recently Pietz [8] has some more general results for the algebras $R(K)$. In his proof, however, he made the assumption that the closure of each component of $\stackrel{K}{K}$, the interior of $K$, has connected complement.

In this note, we show that if $A(U)$ is Dirichlet and $b U_{i}$ has infinite one-dimensional Hausdorff measure, then at least one power of $\left(z-z_{i}\right)^{-n}$ is superfluous in spanning $C(b U)$.

Theorem 1. Let $A(U)$ be a Dirichlet algebra. Assume there is a measure $\mu$ on $b U$ such that $\mu$ annihilates $A(U),\left(z-z_{j}\right)^{-n}$ and $\left(z-z_{i}\right)^{-(n+1)}, j \neq i, n=1,2, \ldots$ while $\int\left(z-z_{i}\right)^{-1} d \mu=1$. Then $b U_{i}$ has finite one-dimensional Hausdorff measure.

Proof. The proof is along the line of [8].

Received by the editors November 25, 1977 and, in revised form, July 7, 1978. 
Theorem 3.1 of [5] implies that each $U_{j}$ is simply connected. A well-known decomposition theorem for Dirichlet algebras (Theorem 1.1 of [5]) gives $\mu=$ $\sum \mu_{j}$ where $\mu_{j}$ is supported on $U_{j}, \mu_{j} \perp A\left(U_{j}\right)$ and $\mu_{j}$ is absolutely continuous with respect to harmonic measure for $z_{j}$ on $b U_{j}$ for each $j$. We may therefore restrict our attention to the pair $\left(\mu_{i}, U_{i}\right)$, which we relabel $(\mu, U)$, and assume $z_{i}=0$.

Let $\phi$ be the Riemann map of $\Delta=\{|z|<1\}$ onto $U$. The map $\phi$ has a measurable one-to-one extension $\phi^{*}$ to a subset of $b \Delta$ of full measure, i.e., $U$ is nicely connected (see, e.g., [1]). Write $\rho$ for the harmonic measure for 0 on $b \Delta$, and $\lambda$ the same for 0 on $b U$. Since $A(U)$ is Dirichlet, there exists $h \in H_{0}^{1}(\rho)=\left\{h \in L^{1}(\rho): \int z^{k} h d \rho=0\right.$ for all $\left.k \geq 0\right\}$ such that $\int f d \mu=\int\left(f \circ \phi^{*}\right) h d \rho$ for all Borel function $f$ on $b U$, by a theorem of Davie ([1], p. 352). Let $w \in H^{1}$ so that $h d \rho=w d z$. Then for any $k, 0<r<1$,

$$
\int_{|z|=r} \phi^{k}(z) w(z) d z=\int_{|z|=r} \phi^{* k}(z) w(z) d z=\delta_{-1, k}
$$

But

$$
\frac{1}{2 \pi i} \int_{|z|=r} \phi^{k}(z) \phi^{\prime}(z) d z=\delta_{-1, k}
$$

Hence $\left(w(z)-\phi^{\prime}(z) / 2 \pi i\right) d z$ annihilates all integral powers of $\phi$, which are uniformly dense on $|z|=r$ by a theorem of Walsh, so that $w(z)=\phi^{\prime}(z) / 2 \pi i$. Therefore $\phi^{\prime} \in H^{1}$. Theorem 3.11 of [3] shows that $\phi$ has an absolutely continuous extension to $|z|=1$ which implies $b U$ has finite one-dimensional Hausdorff measure.

Let $\Psi$ be a homeomorphism of $\varnothing$ to $\varnothing$. Let $V=\Psi(U)$ and $V_{j}=\Psi\left(U_{j}\right)$ for $j=1,2, \ldots$. We say $\Psi$ is singular on $b U$ if $\Psi$ carries a set of full harmonic measure on $b U_{j}$ to a set of zero harmonic measure on $b V_{j}$ for each $j$. In [7], O'Farrell proved that if a compact $K$ has connected complement and $\Psi$ is a homeomorphism singular on $b K$ then $C(b K)$ is the uniformly-closed linear span of $z^{n}$ and $\Psi^{n}, n=0,1,2, \ldots$. By imitating his technique, we can show the following.

THeOREM 2. Let $A(U)$ be a Dirichlet algebra. Let $\Psi$ be a homeomorphism singular on bU such that $A(V)$ is also Dirichlet. Then $C(b U)$ is the uniformlyclosed linear span of $A(U)$ and $A(V) \circ \Psi$.

Proof. Let $\mu$ be a measure on $b U$ which annihilates $A(U)$ and $A(V) \circ \Psi$. Then the measure $\Psi_{\#} \mu$ on $b V$ defined by $\int f d \Psi_{\#} \mu=\int f \circ \Psi d \mu$ annihilates $A(V)$ and $A(U) \circ \Psi^{-1}$. Again the decomposition theorem for Dirichlet algebras gives $\mu=\sum \mu_{j}$ where $\mu_{j}$ is supported on $\bar{U}_{j}, \mu_{j} \perp A\left(U_{j}\right)$ and $\Psi_{\#} \mu_{j} \perp A\left(V_{j}\right)$. Also $\mu_{j}$ and $\Psi_{\#} \mu_{j}$ are absolutely continuous with respect to the harmonic measures for $U_{j}$ and $V_{j}$, respectively. Hence $\Psi_{\#} \mu_{j}=0$, which in turn implies $\mu_{j}=0$ for all $j$ and so $\mu=0$. 
Note that there are Dirichlet algebras $A(U)$ such that $A(V)$ is not Dirichlet for a homeomorphism $\Psi$, e.g., the "string of beads" ([4], p. 145) sets. It would be interesting to know whether Theorem 2 is still true if we replace the singular homeomorphism by an orientation-reversing homeomorphism. This would be a generalized Walsh-Lebesgue Theorem.

These same methods in this note can be used to obtain similar results for a hypo-Dirichlet algebra.

Acknowledgement. The author is grateful to J. Wermer for valuable conversations. The preparation of this paper was supported in part by NSF Grant MPS 74-07035.

\section{REFERENCES}

1. A. M. Davie, Dirichlet Algebras of Analytic Functions, J. Functional Analysis, 6 (1970), 348-356.

2. A. M. Davie, Bounded Approximation and Dirichlet Sets, J. Functional Analysis, 6 (1970), 460-467.

3. P. Duren, Theory of $H^{p}$ Spaces, Academic Press, New York, 1970.

4. T. W. Gamelin, Uniform Algebras, Prentice-Hall, Englewood Cliffs, New Jersey, 1969.

5. T. W. Gamelin and J. Garnett, Pointwise Bounded Approximation and Dirichlet Algebras, J. Functional Analysis, 8 (1971), 360-404.

6. J. Korevaar, and P. Pfluger, Spanning sets of powers on wild Jordan curves, Nederl. Akad. Wetensch. Proc. Ser. A, 77 (1974), 293-305.

7. A. G. O'Farrell, A generalized Walsh-Lebesgue theorem, Proc. Roy. Soc. Edinburgh, to appear.

8. K. Pietz, Cauchy Transforms and Characteristic Functions, Pac. J. Math., 58 (1975), 563-568.

9. J. Wermer, Nonrectifiable simple closed curve, Advanced Problems and solutions, Amer. Math. Monthly 64 (1957), 372.

\footnotetext{
(Current address)

DEPARTMENT OF MATHEMATICS, UNIVERSITY OF AlabAMA, UNIVERSITY, AI.ABAMA 35486

Department of Mathematics, U.C.L.A., L.A., CA. 90024
} 